\title{
Las TIC como estrategia metodológica para desarrollar la expresión oral en adolescentes Kichwa hablantes
}

Fecha de recepción : 12 de junio de 2019 • Fecha de aceptación: 13 de diciembre de 2019 • Fecha de publicación: 13 de enero de 2020

Elizabeth del Rocío Rojas León Unidad Educativa Adolfo Becquer, Ecuador. lizbeth_err111@hotmail.com https://orcid.org/0000-0003-3439-1276

\section{Resumen}

El trabajo aborda los puntos de vista de la autora en torno de las diferentes apreciaciones y cuestionamientos que se han hecho y hacen alrededor de la enseñanza-aprendizaje del idioma inglés en estudiantes kichwa hablantes: ¿Cuáles son las razones para que los estudiantes ecuatorianos mantengan niveles bajos de dominio del idioma inglés? La integración de la tecnología en el aula EFL (English Foreign Language) motivará a los estudiantes de habla kichwa a buscar el aprendizaje y les facilitará el desarrollo de diálogos exitosos y aumentará su dominio del inglés. Los profesores de idiomas necesitan una sensibilidad cultural, así como la capacidad de diseñar aulas virtuales que motiven a los estudiantes a seguir el aprendizaje cultural y los ayude a comunicarse con personas de diferentes culturas. El uso de un aula virtual para la enseñanza del inglés proporcionará herramientas con el que los estudiantes ya están familiarizados, y también será un medio para desarrollar habilidades lingüísticas mientras se comunican y educan a los demás. Además, los estudiantes kichwa hablantes tendrán la oportunidad de desarrollar sensibilidad y apreciación cultural, lo que aumenta su motivación para poder interactuar con otros cuyas culturas son diferentes a las suyas.

\section{Palabras clave: EFL (Inglés como Lengua Extranjera), aula virtual, TIC, Enfoque comunicativo de} idiomas, kichwa hablantes. 


\begin{abstract}
The work addresses the author's points of view around the different opinions and questions that have been asked about the teaching-learning of the English language in Kichwa speakers: What are the reasons for Ecuadorian students to keep low levels in English? The integration of technology into the EFL (English as a Foreign Language) classroom will motivate kichwa speaking students to pursue cultural learning and facilitating them the development of foster successful dialogues and increase their English proficiency. Language teachers need cultural sensitivity as well as the ability to design virtual classrooms that motivates students to pursue cultural learning and helps them communicate with people from different cultures. The use of a virtual classroom for teaching English will provide content that students are already familiar with, and also will be a means to develop language skills while communicating and educating to others. In addition, students will have the opportunity to develop cultural sensitivity and appreciation, adding to their motivation to be able to interact with others whose cultures are different from theirs.
\end{abstract}

Keywords: EFL (English as a Foreign Language), virtual classroom, ICT, Communicative Approach, Kichwa speakers. 


\section{Introducción}

En Ecuador, la educación secundaria, con respecto al nivel del idioma inglés, ha sido insatisfactoria a pesar de una gran variedad de medidas y normativas implementadas para mejorar la adquisición de la lengua inglesa por parte de los estudiantes ecuatorianos. Ecuador se ubica en el puesto cincuenta y cinco de ochenta países con un puntaje promedio de 49.00/100, de acuerdo con el Índice de Aptitud (English Proficiency Index) presentado por la empresa internacional Education First en 2017 (Heredia, 2017).

La Unidad Educativa Comunitaria Intercultural Bilingüe "Gustavo Adolfo Bécquer" es una institución ubicada en la Comunidad "La Compañía Lote 2", de la parroquia de Cangahua, Cayambe, Pichincha. Es una institución de origen indígena, cuya oferta es brindar educación a los estudiantes cayambeños, cuyo primer idioma nativo es el kichwa y su segunda lengua es el español. Ésta brinda una educación basada en el Modelo del Sistema de Educación Intercultural Bilingüe (MOSEIB), que permite fortalecer la instrucción académica de los estudiantes de las nacionalidades y pueblos indígenas. La institución funciona presencialmente, en la jornada matutina y tiene los niveles de Educación Inicial, Básica y Bachillerato.

En la actualidad, en Ecuador existe la presencia de nacionalidades indígenas dentro de su territorio y por ello, se reconoce que el país es intercultural y plurilingüe.

El kichwa en el Ecuador, es un idioma que se extiende en el siglo XV con la expansión inca y se sustenta con las diferentes lenguas que existían en los pueblos intervenidos de la época. A la llegada de los españoles fue el idioma que se utilizó para evangelizar a los pueblos originarios de la Sierra y parte de la Amazonía; sin embargo, este idioma se preserva como la forma de comunicación al interior de las familias y comunidades. (Ministerio de Educación, 2016, p.4)

La nacionalidad Kichwa se constituye en dieciséis pueblos que se ubican la mayor parte en la región Sierra y el norte de la región amazónica. No obstante, es importante destacar que la población migrante kichwa se encuentra en las ciudades principales del Ecuador: Quito, Guayaquil, Cuenca y Galápagos, formando más ambientes sociales interculturales (Ministerio de Educación, 2016).

Por lo tanto, el objetivo planteado para la educación intercultural es crear sociedades democráticas, inclusivas y libres de racismo y etnocentrismo. En los años ochenta, las organizaciones de los pueblos indígenas reclamaban una participación lingüística y cultural igualitaria de las minorías. Desde entonces, lo que se pretende lograr son sociedades pluriculturales, en las que todos los ciudadanos contribuyan de forma activa a la convivencia pacífica en condiciones de igualdad de derechos. Para el éxito de este propósito, todas las culturas implicadas deben ser capaces de aceptar diversos procesos de aprendizaje (LED, 2013).

Obsérvense, vistos desde el análisis crítico de la autora, algunos de estos cuestionamientos: 


\section{Desarrollo}

\section{1. ¿El kichwa en el Ecuador, un idioma fortalecido o inutilizado?}

En el año 2008, la Constitución Política del Ecuador mediante la Asamblea Nacional, en el artículo 6, literal 1, admite la presencia de etnias indígenas dentro de Ecuador y por ende, que es un pueblo intercultural y plurilingüe. El español es el idioma oficial del Ecuador, junto con el kichwa y shuar que son lenguas oficiales de relación intercultural. Los otros idiomas ancestrales permanecen de uso oficial para los pueblos indígenas en las zonas donde residen y de acuerdo a la ley (Sánchez, 2017).

En Cayambe se han integrado los programas curriculares de manera progresiva a los estudiantes kichwa hablantes para brindarles una igualdad de oportunidades en el ámbito profesional pero conservando una sociedad ecuatoriana intercultural, que valore la diversidad lingüística y cultural de nuestro país Ecuador.

Con este antecedente, la Subsecretaria de Educación implementa en los distritos educativos el currículo para la enseñanza-aprendizaje de la Lengua Extranjera (inglés), y lo socializa con los docentes ecuatorianos para impartirlo a los estudiantes kichwa hablantes como una asignatura optativa en la malla curricular. Esta acción educativa, implica el reconocimiento de los pueblos indígenas, la valoración de nuestras raíces, el respeto y fortalecimiento a la educación intercultural de Ecuador y por ello a una instrucción académica acorde a las necesidades actuales.

\section{2. ¿Cuáles son las habilidades del idioma inglés que desarrollan los estudiantes kichwa hablantes?}

Las cuatro habilidades principales ingleses que desarrollan los estudiantes kichwa hablantes son: escuchar, leer, hablar y escribir se describen en dos grupos: "Productivas" y "ReceptivasInterpretativas". Las habilidades tradicionales se clasifican en "activas" (hablar y escribir) y "pasivas" (escuchar y leer). Esta terminología es claramente engañosa, ya que no podemos decir que los oyentes y los lectores no hagan nada en absoluto cuando intentan entender un texto oral o escrito. Esta es la razón por la cual "productivas" (en el sentido de que "produces" algo cuando hablas o cuando escribes) y "receptivas-interpretativas" (recibes un mensaje oral o escrito e interpretarlo) parecen ser términos más apropiados (Strobelberger K. , 2012).

Por un lado, el registro oral se comparte hablando y escuchando, mientras que el escrito es común para escribir y leer. Por ello, hablar y escribir se ven afectados por cuestiones metodológicas similares debido a que son "productivas", mientras que lo mismo puede decirse de escuchar y leer sobre la base de su carácter "receptivo" (Strobelberger K. , 2012).

Según Wallace, Stariha y Walberg (2004), los estudiantes kichwa hablantes necesitan saber cómo los hablantes del idioma se diferencian unos de otros y cómo las circunstancias particulares requieren diferentes formas de hablar. Se puede aprender también cómo los estilos del habla afectan a los oyentes. Por lo tanto, la velocidad a la que hablan, el volumen y la precisión de 
pronunciación pueden diferir sustancialmente de una situación a otra. Es útil que los estudiantes sepan que el discurso debe diferir en formalidad, como cuando se habla con un juez, un maestro, un padre o un compañero de juegos.

También pueden beneficiarse de aprender sobre las diferencias entre varios dialectos. Las materias en el plan de estudios y los ejemplos de los medios pueden proporcionar ocasiones para diferentes formas de discurso. Las presentaciones orales pueden derivarse de poemas, historias, artículos de periódicos y revistas, así como informes científicos. La actuación dramática, la observación de sketches y obras de teatro pueden proporcionar la mejor oportunidad para ver cómo el carácter y las circunstancias afectan el habla.

Ante esta situación, las unidades educativas fiscales ecuatorianas están en la obligación de responder asertivamente a los requerimientos de la sociedad y al entorno social, económico y cultural en las que éstas se desarrollan (Chérrez, 2014). Por ello, la Dirección Nacional de Currículo, como parte de la política educativa, ha diseñado un nuevo currículo de Lengua Extranjera (Inglés), que responda a las necesidades de la realidad ecuatoriana actual pero que se adapte al Marco Común Europeo de Referencia para las Lenguas (MCER). Esta iniciativa está orientada a velar por la transparencia del sistema educativo, claramente desatendido durante décadas, lo cual se evidencia en la existencia de múltiples brechas en aspectos nodales de la calidad de la Educación Básica en el ámbito académico, investigativo y tecnológico.

\section{3. ¿Integración de la tecnología; una estrategia metodológica que desarrolla la} expresión oral del idioma inglés?

Se estima que en el Ecuador, la Educación durante décadas, se ha enmarcado en procedimientos tradicionalistas y controlados, donde el docente es el único protagonista en impartir conocimiento mediante clases magistrales, dejando de lado los nuevos paradigmas y metodologías que integran el uso de las Tecnologías de la Información y Comunicación (TIC), y los aprendices contribuyen en la adquisición del conocimiento (Chérrez, 2014). La razón principal se debe a la falta de políticas gubernamentales claras y la escasa inversión que el Gobierno daba a la Educación, causando una brecha digital entre nuestra sociedad y las sociedades aledañas en Latinoamérica (Chérrez, 2014).

Por lo tanto, el aprendizaje a través del uso de la tecnología es muy beneficioso, y contribuye aún más con el aprendizaje de idiomas en las escuelas públicas de Ecuador porque permite dotar a los jóvenes de destrezas y valores que son indispensables para enfrentar los desafíos que la conectividad está causando (Chérrez, 2014).

Más aún, para brindar calidad en la educación actual, la tecnología se ha convertido en un recurso de enseñanza que promueve la gestión de la clase; es decir, el docente puede utilizar las TIC como un medio para la producción de material didáctico, planificación macro, meso y micro curricular, así como también, la presentación de información en diferentes herramientas tecnológicas atractivas para el educando (Educación, 2016).

De acuerdo a Salinas (2004) quien sugiere: "Para que tanto las instituciones existentes como 
las que están naciendo puedan responder verdaderamente a este desafío, deben revisar sus referentes actuales y promover experiencias innovadoras en programas curriculares apoyados en las TIC" (p. 2). En este aspecto, las instituciones se enfrentan a dificultades asociadas a la infraestructura ya que no cuentan con espacios adecuados para laboratorios de computación y éstos no disponen de los computadores suficientes con internet, por ello las autoridades educativas deben coordinar acciones en beneficio de los educandos para dotar a las instituciones tecnología de punta y ofrecer calidad educativa.

La Educación Virtual es un factor transformador en la educación pública, un instrumento para mejorar su cobertura, calidad, pertinencia y equidad de acceso, porque está sirviendo a una población cada vez mayor de estudiantes, más diversificada social y culturalmente, más dinámica y cambiante. Adicionalmente, los estudiantes actuales, especialmente aquellos menores de treinta años tienen una habilidad innata con la tecnología, ya que forma parte de su vida y es por este motivo que los docenes debemos innovar nuestra tarea, generando nuevos entornos de enseñanza-aprendizaje y aprovechando todo el potencial tecnológico que tenemos a nuestro alcance, con el fin de mejorar la calidad de la educación ecuatoriana.

Sin embargo, al implementar las herramientas tecnológicas en las zonas rurales del país, las instituciones educativas deberán enfrentarse a dificultades como docentes que no tienen conocimiento de Informática, desafíos de crear recursos multimedia o fracasos al no poder controlar la información disponible para los educandos, pero lo importante es usarlos como oportunidades de aprendizaje y resolución de problemas para evaluar e involucrar a los estudiantes en esta travesía de la educación virtual.

Como sugiere Chamorro (2015) las necesidades de la juventud ecuatoriana establecen el inicio para el diseño de un aula virtual. Las interpretaciones de estas se plasman en la Ley 115 de 1994 , Ley 715 de 2001, Plan Decenal de Educación y demás decretos y resoluciones que regulan las leyes, así como en las interpretaciones de los Objetivos del Milenio y las Metas 2021.

De acuerdo a Hernández (2014) el uso de las TIC permite algunas ventajas en el campo educativo.

Es un proceso complejo, dando oportunidad al cerebro de trabajar de diferentes maneras e integralmente, incorporando todas las dimensiones como: cognición, reflexión, crear, sintetizar y verbalizar. Este tipo de aprendizaje además favorece el compromiso del alumnado con su realidad incitándole a una participación más activa, crítica y responsable en la que los estudiantes tienen la oportunidad de compartir experiencias y debatir, favoreciendo el desarrollo de una mayor capacidad para el respeto y la aceptación de otros puntos de vista en busca de la consecución de metas comunes. (p.86)

Se ha mostrado un gran interés por la integración de herramientas tecnológicas a través de un aula virtual para la enseñanza-aprendizaje de la Lengua Extranjera (Inglés) con los estudiantes kichwa hablantes de Octavo Grado, con el objetivo de desarrollar las habilidades comunicativas de los estudiantes. Según Equipo Técnico Inglés (2016), los Estándares de Competencias proyectan niveles que deben ser alcanzados por los aprendices, con el propósito de contribuir 
a tener ciudadanos y ciudadanas capaces de comunicarse, con estándares internacionalmente comparables.

Según expresa la tesis de Castro (2005) que se enfoca a las orientaciones curriculares sobre las TIC y la enseñanza de la lengua inglesa, asegura que los estudiantes deben tener conocimientos básicos de Ofimática para ser capaces de trabajar con plataformas virtuales, creando de esta manera un ambiente motivador para los aprendices en su contexto educativo, teniendo una gran acogida en el proceso de enseñanza-aprendizaje. El profesor y el alumno tienen una amplia gama de actividades para la adquisición de las habilidades principales: escuchar, hablar, escribir y leer; mediante el uso de la tecnología en el campo docente y la disponibilidad del equipo tecnológico.

Además, en otra investigación científica: Silvia (2006) plantea el enfoque sobre una perspectiva no sólo didáctica y lingüística sino también psicolingüística y sociolingüística, donde tuvo un gran éxito el uso de canciones en el aula, siendo un gran recurso de motivación para los estudiantes porque incentivan su esfuerzo en el aprendizaje del idioma inglés. Aún más, este recurso para la enseñanza de idiomas fue muy eficaz, con el fin de trabajar el acento y la entonación, reforzar vocabulario aprendido en clase, ayudando a mejorar la habilidad de escuchar, y también mejorando la autoestima del estudiante para expresar sus sentimientos y sensaciones.

En la búsqueda de modelos de integración, se manifiesta la necesidad de investigar los procesos que ocurren durante el aprendizaje con el empleo de la tecnología. Riding \& Bucle (2003) reconocieron la integración del aprendizaje basado en las TIC como fundamental en la práctica educativa. Casi dos decenios más tarde, la integración curricular de las Tecnologías de la Información y Comunicación es aún una prioridad de la informática educativa ya que las zonas rurales todavía no cuentan con este beneficio por su situación geográfica de difícil acceso.

\section{4. ¿La tecnología en el aprendizaje de idiomas; un enfoque integrador o individualista?}

La tecnología en el aprendizaje facilita un enfoque integrador de la educación fiscal ecuatoriana, un canal para incrementar su alcance, calidad, pertinencia social y equidad en la accesibilidad, puesto que está disponible para una población cada vez mayor de estudiantes, más diversificada social y culturalmente, más dinámica y cambiante (Senplades, 2017) .

Además, los estudiantes de la generación actual, en especial los aprendices menores de treinta años, quienes tienen una habilidad innata con la tecnología, porque forma parte de su vida cotidiana y es por ello que los docentes de vocación deben innovar su tarea, creando nuevos ambientes de enseñanza-aprendizaje y aprovechando todo el potencial tecnológico que tienen a su alcance, con el fin de mejorar la calidad de la educación ecuatoriana.

El docente debe asumir la responsabilidad de estructurar claramente las actividades de la manera más adecuada posible para completar con éxito un conocimiento. El aprendizaje en las etapas iniciales se facilita si los estudiantes atienden una tarea a la vez. El docente alienta la iniciativa e independencia del alumno, pero no permite que los alumnos forcejeen en silencios incómodos. Los estudiantes necesitan tiempo de reflexión silencioso para aprender. Los estudiantes aprenden 
mejor cuando tienen opciones que les ofrezcan práctica, de esta manera, ellos desarrollan una sabiduría interna sobre dónde necesitan trabajar. Si se sienten en control, pueden asumir una mayor responsabilidad por su propio aprendizaje. Los estudiantes deben aprender a discriminar, por ejemplo: percibir las similitudes y diferencias entre las formas del idioma a aprender (Larsen \& Freeman, 2010).

En general, según Mejía (2012) las aulas virtuales son un eje primordial en la transformación de la educación y los constantes cambios que están surgiendo en los entornos de aprendizaje virtuales. En los últimos años, las aulas virtuales funcionan eficazmente en la asignación de tareas, foros, seguimiento académico de los estudiantes, evaluaciones en línea, entre otros., donde tanto alumnos como profesores e investigadores pueden acceder sin limitaciones de tiempo y espacio, produciendo cambios en la no utilización de materiales impresos, guías de estudios, de trabajo académicos.

Finalmente, de acuerdo con Tessio y Di Stefano (2015) el Ministerio de Educación reconoce que el objetivo principal del actual diseño del currículo de inglés es ayudar a los estudiantes a desarrollar sus habilidades de lenguaje comunicativo a través de la consideración de los siguientes principios:

- El lenguaje es un sistema que facilita la expresión y la transmisión de significado.

- La función principal del lenguaje es la interacción y la comunicación.

- La estructura del lenguaje refleja sus usos funcionales y comunicativos.

\section{Conclusiones}

- En las instituciones cayambeñas donde predomina la existencia de estudiantes kichwa hablantes es primordial dotarles de tecnología de punta para usarlos como oportunidades de aprendizaje significativas que incluyan el proceso formativo y la evaluación, involucrando a los estudiantes en este beneficio de la educación virtual actual.

- Es fundamental que los docentes realicen procesos adecuados al instante de diseñar, elaborar y ejecutar un aula virtual, con el objetivo de garantizar la calidad en el desarrollo de las habilidades comunicativas del idioma inglés en los estudiantes. Kichwa hablantes.

- La enseñanza debe ser enfocada con propósitos formativos, con una intencionalidad definida, con criterios claros para contribuir a la formación de los estudiantes, que permita una mayor coherencia entre la teoría y la práctica sin caer en el discurso complejo y abstracto.

- Las aulas virtuales benefician la labor docente en cuanto a dónde y cómo se produce el desarrollo de la compresión oral, así como también, introduce cambios en los roles de profesores y estudiantes kichwa hablantes de Octavo Grado, para utilizar al máximo los beneficios de las aulas virtuales y aplicar herramientas de comunicación y de trabajo colaborativo tanto para los futuros docentes como para los estudiantes en actividad. 


\section{Referencias Bibliográficas}

Castro, P. (2005). Estrategia de integración de la alfabetización electrónica a la enseñanza-aprendizaje del inglés con fines específicos en la carrera de ingeniería agronómica. Obtenido de www.biblioteca.uma.es/ bbldoc/tesisuma/16853805.pdf

Chérrez, E. (2014). Universidad Técnica de Ambato. Obtenido de El B-learning como estrategia metodológica para mejorar el proceso de enseñanza-aprendizaje de los estudiantes de inglés: https://es.scribd.com/ document/372830145/T35913PDF-Ensenanza-del-idioma-Ingles-E-Prints-Complutense-Universidad

Educación, I. I. (2016). Programa de Mejoramiento del Sistema Educativo. Obtenido de unesdoc.unesco.org/ images/0015/001507/150785s.pdf

Equipo Colombia Bilingüe. (2016). Obtenido de eprints.ucm.es/29610/1/T35913.pdf

Equipo Técnico Inglés. (2016). The English Language Learning Standards. Obtenido de https://educacion.gob. ec/wp-content/plugins/.../download.php?id...

Heredia, V. (8 de Noviembre de 2017). Sociedad. Ecuador alcanzó el puesto 55 de 80 en idioma inglés, según EF English Proficiency Index.

Hernández, E. (2014). El B-learning como estrategia metodológica para mejorar el proceso de enseñanza-aprendizaje de los estudiantes de Inglés. Obtenido de eprints.ucm.es/29610/1/T35913.pdf

Larsen, D., \& Freeman. (2010). Techniques and Principles in Languages Teaching. Oxford: Oxford University Press.

LED. (21 de Marzo de 2013). Estrategia para la Educación Intercultural Bilingüe (EIB) (2013 - 2017) . Obtenido de Servicio de Liechtenstein para el desarrollo: www.led.li/fileadmin/user_upload/Downloads/2013_Estrategie_EIB.pdf

Mejía, P. (Noviembre de 2012). EI AULA VIRTUAL EN EL DESARROLLO DE LA COMPRENSIÓN ORAL DEL IDIOMA INGLÉS. Obtenido de https://dialnet.unirioja.es/descarga/articulo/5968471.pdf

Ministerio de Educación. (2016). Lengua Kichwa. Obtenido de https://educacion.gob.ec/wp.../10/Asignatura-Optativa-Lengua-Kichwa-LL-3BGU.pdf

Otavalo Intercultural. (04 de Septiembre de 2018). Promueven la educcación intercultural bilingüe. La Hora, pág. 1.

Salinas, J. (2004). Innovación docente y uso de las TIC en la enseñanza. 
Sánchez, C. (1 de Abril de 2017). ResearchGate. Obtenido de Políticas Lingüíticas del Ecuador en relación al idioma kichwa: https://www.researchgate.net/publication/318278284_POLITICAS_LINGUISTICAS_DEL_ ECUADOR_EN_RELACION_AL_IDIOMA_KICHWA

Senplades. (11 de Octubre de 2017). Ministerio de Educación. Obtenido de Plan Nacional de Desarrollo 20172021.Toda una Vida: www.planificacion.gob.ec/wp.../2017/10/PNBV-26-OCT-FINAL_OK.compressed1.pdf

Silva, M. (2006). EL USO DE CANCIONES DE MÚSICA. Obtenido de eprints.ucm.es/29610/1/T35913.pdf

Strobelberger, K. (2012). Classroom Discourse in EFL Teaching: A Cross-cultural Perspective.

Tessio, N., \& Di Stefano, A. (2015). Dialnet. Obtenido de Nuevas herramientas para la evaluación a distancia, hacia un nuevo rol docente: https://dialnet.unirioja.es/descarga/articulo/5452162.pdf

Wallace, T., Stariha, W., \& Walberg, H. (01 de 11 de 2014). ResearchGate. Obtenido de Teaching speaking, listening and writing: https://www.google.com/url?sa=t\&rct=j\&q=\&esrc=s\&source=web\&cd=1\&cad=rja\&uac$\mathrm{t}=8 \&$ ved=2ahUKEwjK6_2HuNDgAhUSEawKHSOLCfYQFjAAegQIBhAB\&url=https\%3A\%2F\%2Fwww. researchgate.net\%2Fpublication\%2F44835944_Teaching_speaking_listening_and_writing\&usg=AOvVaw0-YjoQu66Dqu 